\title{
New coordinate-tracking detector on drift chambers for registration of muons in near-vertical EAS
}

\author{
V.S. Vorobev ${ }^{a, *}$, E.P. Khomchuk ${ }^{a}$, R.V. Nikolaenko ${ }^{a}$, A.A. Petrukhin ${ }^{a}$, I.Yu. Troshin ${ }^{a}$ \\ and E.A. Zadeba ${ }^{a}$ \\ a National Research Nuclear University MEPhI (Moscow Engineering Physics Institute), \\ Kashirskoe highway, 31, Moscow, Russia \\ E-mail: VSVorobev@mephi.ru
}

The new coordinate-tracking detector ProtoTREK was developed in the Experimental complex NEVOD, MEPhI for the study of near-vertical extensive air showers. The installation consists of two planes of drift chambers, seven in each, and has an effective area of $13 \mathrm{~m}^{2}$. The registration system of the new detector gets trigger from scintillator counters and uses the TDC that has been specially designed for this detector. It is based on FPGA Altera Cyclone V and has GPS synchronization with the joint triggering system of the experimental complex. A deep learning approach is used to reconstruct multi-muon events registered by ProtoTREK. The paper presents the design of the detector and first experimental results.

37th International Cosmic Ray Conference (ICRC 2021)

July 12th-23rd, 2021

Online-Berlin, Germany

\section{"Presenter.}




\section{Introduction}

In MEPhI the new large-scale detector TREK based on multiwire drift chambers is being developed [1]. It is designed for registration of near-horizontal EAS of ultra-high energies. Joint operation of Cherenkov water calorimeter NEVOD [2] and TREK (Fig. 1) will allow to solve so-called "muon puzzle", an excess of cosmic ray muons compared to simulations [3]. The first prototype of the full detector, Coordinate-Tracking Unit based on Drift Chambers (CTUDC), was made in 2017 [4]. It had two parallel planes of 8 chambers (also seen in Fig.1). The goals of CTUDC were investigation of possibilities of joint operation of drift chambers with other installations of the experimental complex NEVOD, registration of events with high density of particles, development of reconstruction methods, DAQ electronics etc. But this detector allows to reconstruct only one projection of zenith angle of particle tracks. Therefore, the new detector based on drift chambers has been developed. It was designed as prototype of TREK and called ProtoTREK. After the launch of the TREK operation of the prototype will complete the data of the full detector in the range of zenith angles from $30^{\circ}$ to $60^{\circ}$.

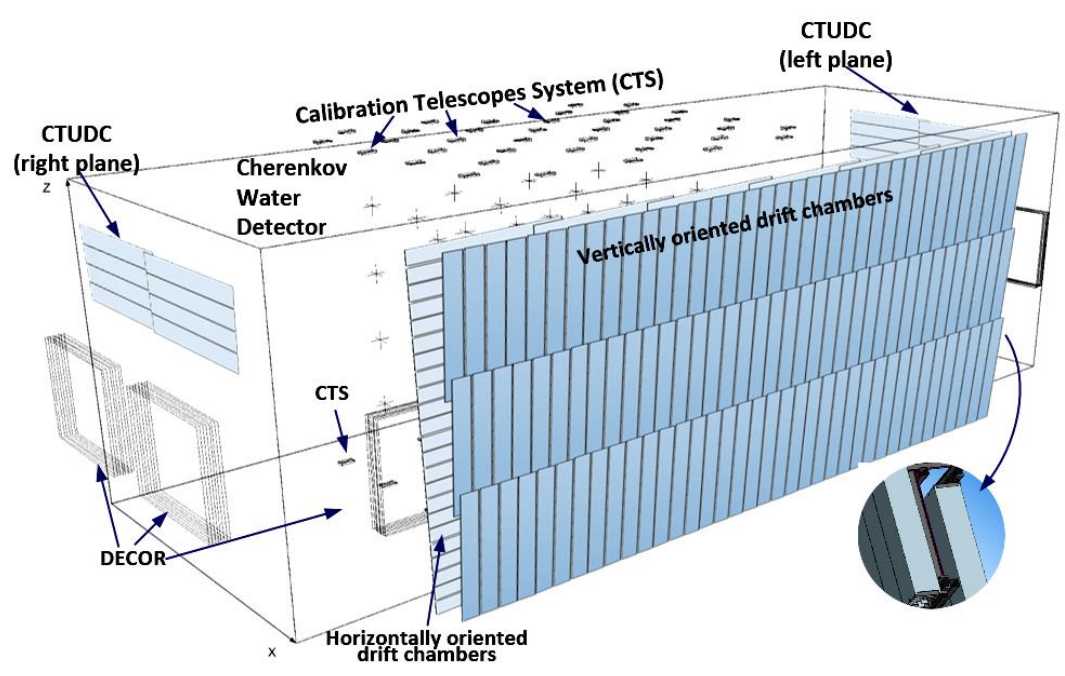

Figure 1. Experimental complex NEVOD and coordinate-tracking detector TREK.

\section{ProtoTREK}

The drift chambers used in TREK were developed by Institute of High Energy Physics for neutrino experiments at U-70 accelerator [5]. The size of the chamber is $4000 \times 508 \times 112 \mathrm{~mm}^{3}$. Fig. 2 shows the cross section of the drift chamber. It has the array of fieldforming wires, 10 cathode wires and 4 signal wires. The signal wires are located near the center of the chamber and staggered from it to determine which side the particle passed. Chambers are filled by gas mixture of $94 \% \mathrm{Ar}$ and $6 \% \mathrm{CO}_{2}$. The maximum drift time is about $6 \mu$ s with constant velocity.

The ProtoTREK has 2 non-parallel planes of 7 chambers in each. The scheme of the installation is shown in Fig. 3. Each plane is included at an angle of $3.5^{\circ}$ to the horizon. It copies chambers orientation in TREK, where they are rotated to overlap each other's dead zones (it is shown in Fig.1). There are two square scintillator detectors $\left(1 \mathrm{~m}^{2}\right.$ each) above and below coordinate planes. They are connected to the coincidence circuit that acts as a trigger for ProtoTREK registration system. 


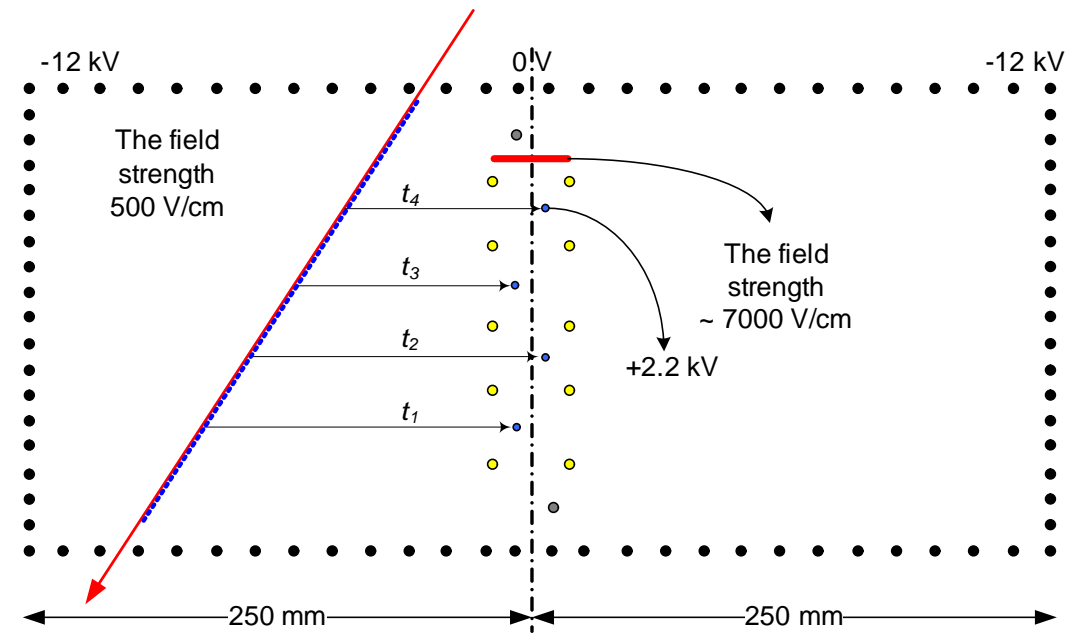

Figure 2. Cross section of the drift chamber (not in a scale).
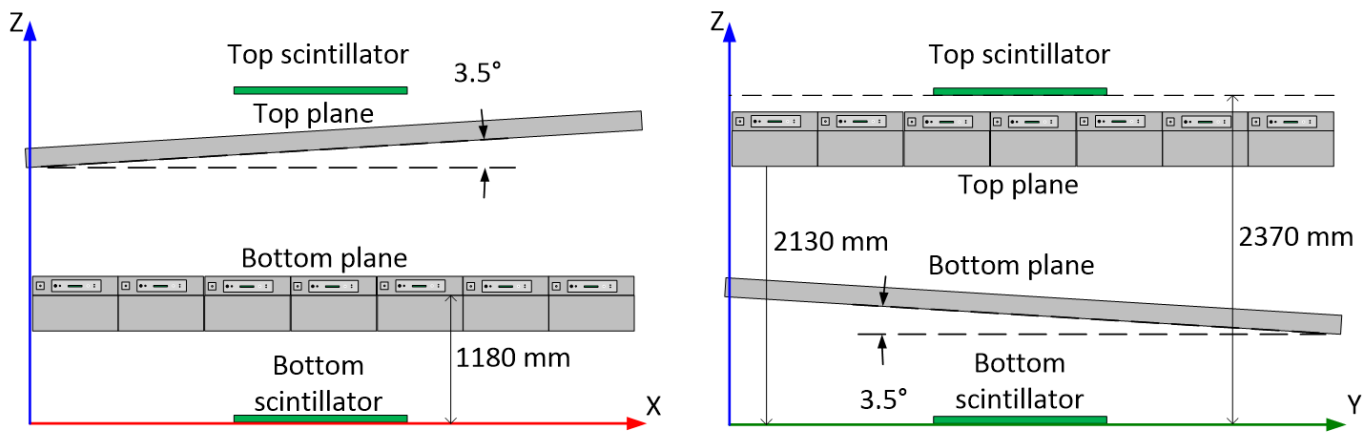

Figure 3. Orientation of ProtoTREK coordinate planes.

The new 32-channel time-to-digital converter (TDC) was developed in MEPhI for multiwire drift chambers [6]. It is based on FPGA Altera Cyclone V [7]. One TDC allows to operate 8 drift chambers. The TDC recieves signals from shaper-amplifiers AMP-4 (installed at each chamber) in LVDS levels at a rate of $200 \mathrm{MHz}$ and writes information about them into the RAM buffer $(4096 \times 32$ bit words) within FPGA. The bit number corresponds to a certain signal channel. Value «1» of a bit corresponds to a signal presence. The word number corresponds to time from the start of a matching window. The time step is $5 \mathrm{~ns}$. The width of the matching window is $20 \mu$ s (such large value is still used for debugging, and further it will be limited to 8 $\mu \mathrm{s})$.

The TDC has operation system Linux on the board. It allows to control the TDC and get information from it by server-client system. After the arrival of the trigger from coincidence system the client on the board collects signals from measuring channels within matching window. Then it forms a network package with main information about an event and sends it to the server.

The package contains information about event time, it is determined as follows. After the launch of the server software located on the central PC it receives the initial UTC timestamp from the GPS antenna connected via the Arduino board [8]. This integer time, expressed in seconds, is sent to the client software. After that, every second a pulse-per-second (PPS) signal is sent from the GPS antenna to the both TDC boards of ProtoTREK. After each PPS the time expressed in seconds on the TDC is increased by one. This allows us to accurately determine the 
time of the event in seconds. The internal generator of the board is used to determine the subsecond time value (Fig. 4). The generator operates at $50 \mathrm{MHz}$. It means that $n_{g}=50$ million generator signals are expected between PPS signals. At the random moment of trigger arrival the number $n_{t}$ of signals is got from the generator from the last PPS. The ratio $n_{t} / n_{g} \times 1 \mathrm{sec}$ is the time of arrival of the trigger $t_{\text {trig }}$ from the moment of registration of the last PPS. Summing up the time in seconds and this ratio, we get the trigger arrival time with an accuracy of $20 \mathrm{~ns}$.

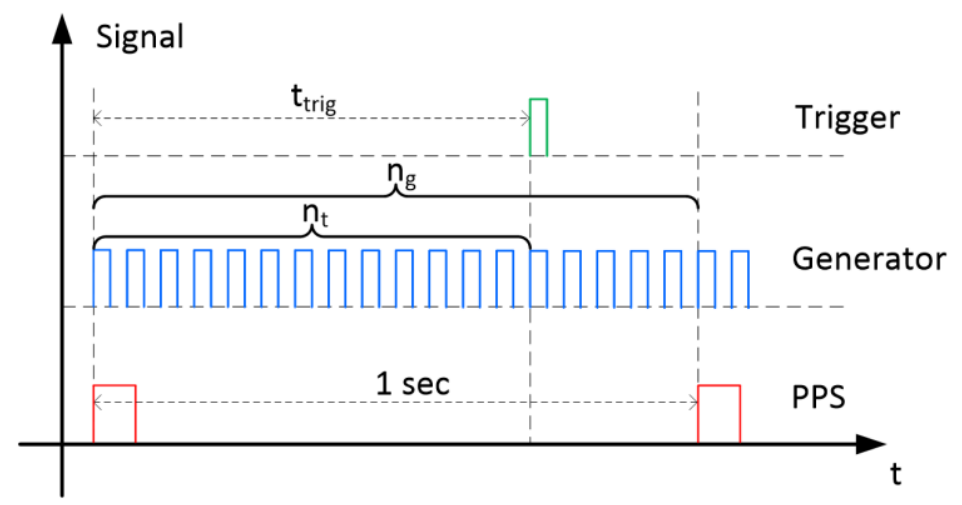

Figure 4. Determining the subsecond value of the trigger arrival time.

One TDC services one plane of the installation. Two TDC are synchronized by GPS: if the events at both boards lie within $1 \mu$ s interval they are merged to a single ProtoTREK event. In fact, the difference of times at TDCs for a same event rarely exceeds $100 \mathrm{~ns}$.

For operation of the drift chambers 3 power circuits are required. Low-voltage power supply is provided by AKIP-1115, it provides $\pm 5.5 \mathrm{~V}$ for the operation of the shaper-amplifiers. For signal wires of drift chambers $+2.2 \mathrm{kV}$ is required, and for field-forming ones $-12 \mathrm{kV}$. That is provided by programmable high-voltage source, developed by High-voltage systems laboratory, Dubna.

To provide the chambers with the required gas mixture, a programmable supply system is used. Due to the need to flow a large volume of mixture through the drift chambers, a 500 liter cryocylinder is used as a reservoir for liquid argon. With a constant flow of 100 liters of gaseous argon per hour, its volume will be enough for 4 months of operation. The carbon dioxide is stored in 401 gas cylinders as its consumption is small. The gas speed is controlled by digital flow meters for each component of the mixture. The planes are connected to the gas system separately, and within each plane chambers are connected continuously one-by-one.

\section{Method of event reconstuction}

Three analytic reconstruction methods for the drift chambers were used for CTUDC [9]. But the main disadvantage is that these methods cannot take into account the presence of afterpules. Their existence is associated with geometry of electron clouds. Due to the fact that the width of the electron cloud is not equal to zero, it can cause more than one signal on the channel. The presence of such signals leads to fake reconstructions.

To solve this problem, the new approach of analysis based on deep learning techniques is developed. To reduce afterpulses we designed a filter based on convolutional neural networks [10]. We can present waveforms from a chamber signal channel in a form of matrix with size $4 \times 600$ (Fig. 5). Each row corresponds to a $10 \mathrm{~ns}$ interval with a total time interval of $6 \mu$ s. Value 
«1» of a bit corresponds to signal presence, and value «0» corresponds to absence of it. Using such a matrix as an input of a network and obtaining matrix with such size as an output we can exclude afterpulses from the matrix.

\begin{tabular}{|l|l|l|l|l|l|l|l|l|l|l|l|}
\hline 0 & 0 & 0 & 1 & 1 & 1 & 0 & 0 & 0 & 0 & 0 & 0 \\
\hline 0 & 0 & 0 & 0 & 1 & 1 & 1 & 0 & 0 & 0 & 0 & 0 \\
\hline 0 & 0 & 0 & 0 & 0 & 1 & 1 & 1 & 0 & 0 & 0 & 0 \\
\hline 0 & 0 & 0 & 0 & 0 & 0 & 1 & 1 & 1 & 0 & 0 & 0 \\
\hline
\end{tabular}

Figure 5. Representation of data from a drift chamber in the form of a binary matrix.

The experimental data also can contain signals from secondary particle tracks that also lead to fake reconstruction. The new method based on recurrent LSTM neural network [11] is designed. The idea is based on the work [12]. We can also use the same matrix as an input. One signal is fixed on the first channel, and the rest are excluded from the image. The network analyzes the signal channels sequentially. It leaves only those signals on the other three channels that correspond to the fixed hit on the first. Then, a simple reconstruction through the four points is carried out. At the next iteration another signal is fixed on the first channel, etc. In other words, this network separates signals to different tracks. An example of operation of both networks is presented in Fig. 6. The middle waveform is input matrix with all signals. The bottom waveform is output of networks. Colors of signals are result of the second network operation. Tracks reconstruction is shown on the top.

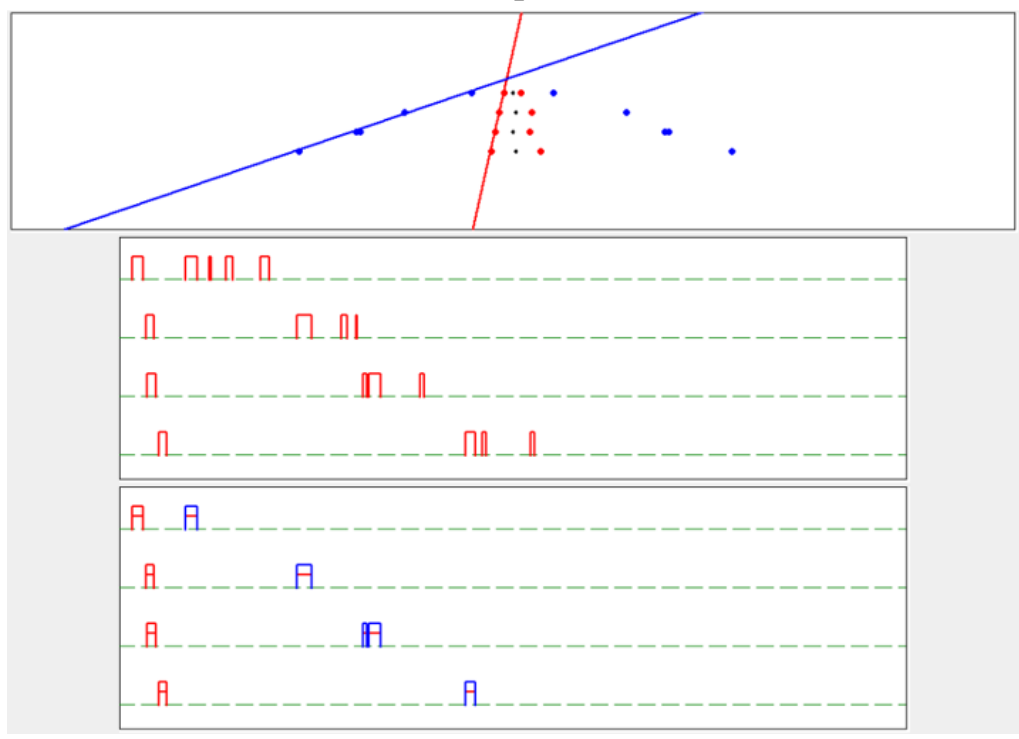

Figure 6. Reconstruction of experimental event by deep learning methods.

Both networks were trained on experimental data of CTUDC. The filter allows to exclude up to $85 \%$ of all afterpulses. The recurrent network showed an error in multiplicity determination less than $10 \%$ even in the most dense events registered by CTUDC.

\section{Reconstruction of events registered by ProtoTRE}

Using the new approach we reconstructed and analyzed events detected by ProtoTREK in a time interval between 30.04.2021 and 04.06.2021. The live operating time of the installation for this period is 706 hours. 
Fig. 7 shows distribution of events in multiplicity of all reconstructed charged particle tracks by each plane of the detector. For events with more than 100 particles per plane, the signals from the tracks merge so much that event reconstruction is not reliable.
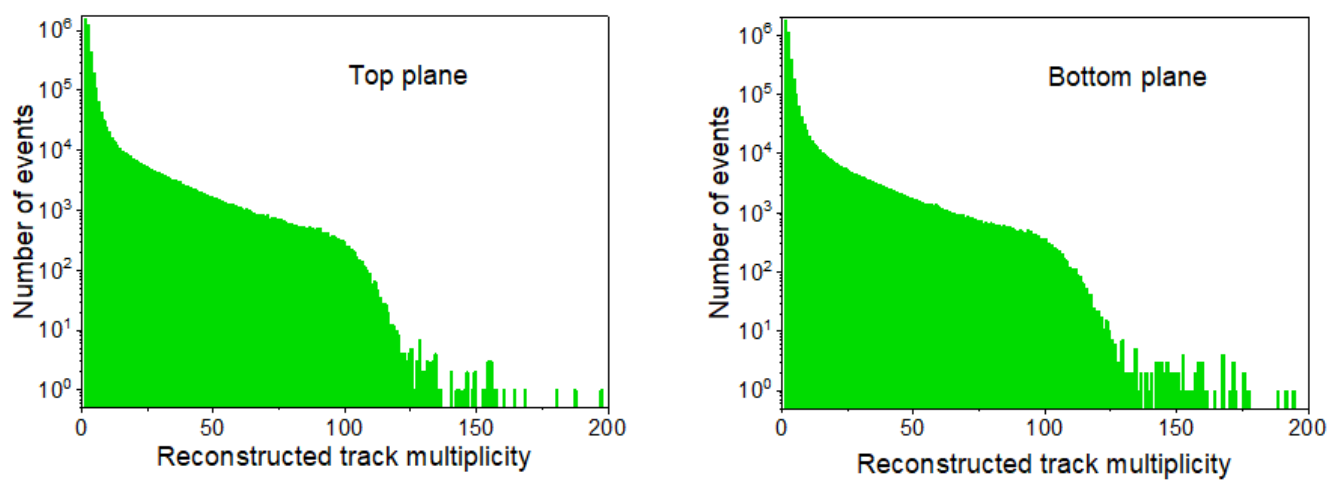

Figure 7. Distributions of events in multiplicity for coordinate planes.

Among these events, one can single out those in which parallel tracks are observed. We assume that these parallel tracks correspond to muons. The following criteria are used to highlight these events:

1) Parallel tracks that lie in an angular interval of $\pm 5^{\circ}$ is considered.

2) The multiplicity of parallel tracks on each plane must be three and more.

3) The portion of parallel tracks on each plane must be more than $50 \%$.

Figure 8 shows distributions in multiplicity for parallel tracks. The maximum number did not exceed 45 . The maximum density in these events is 3.5 particles per square meter.

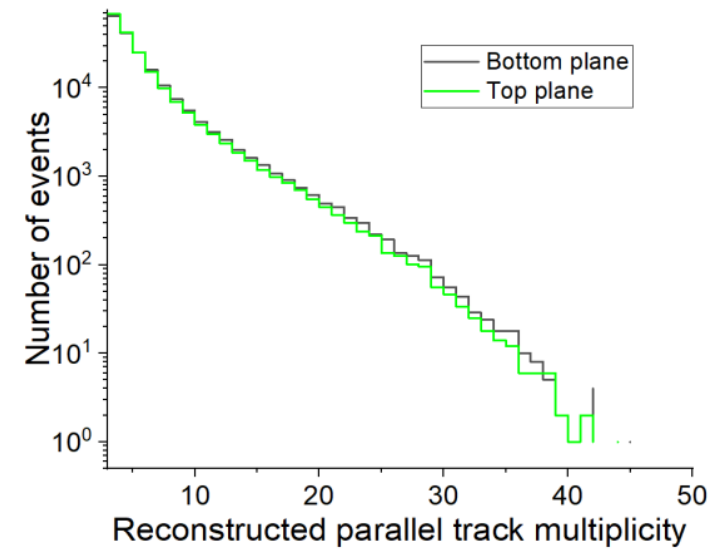

Figure 8. Distributions of events in multiplicity of parallel tracks.

Figure 9 shows the angular distributions of these events. Events of the azimuth angle distribution correspond to zenith angles $\leq 15^{\circ}$. The reasons of the deviations of the azimuthal distribution from the uniform one are the influence of closest surrounding buildings of the University and performance of scintillator detectors. 

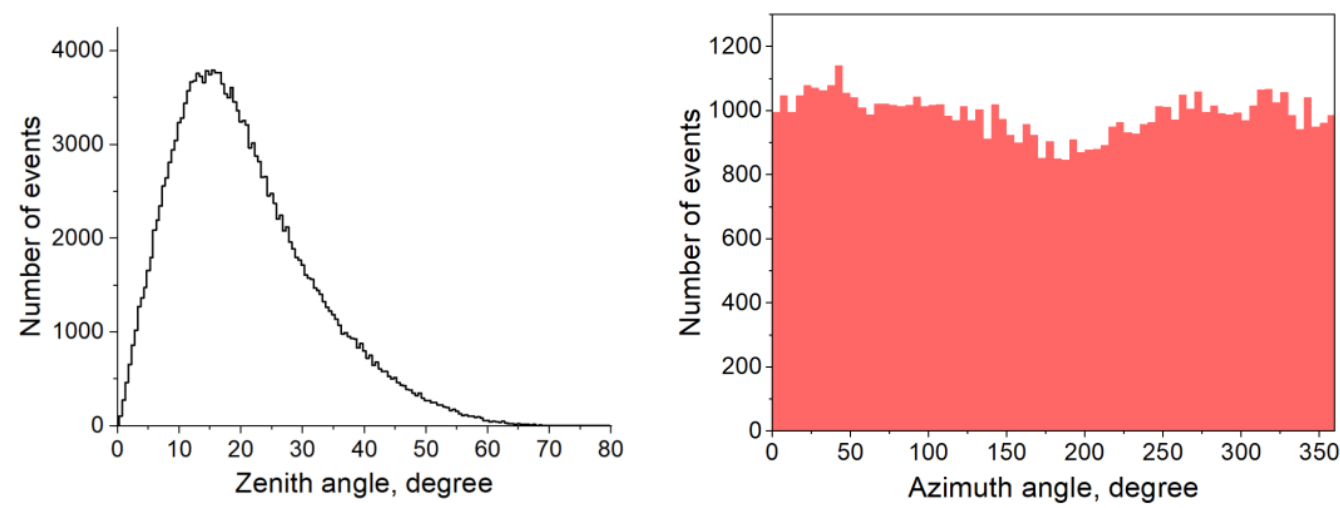

Figure 9. Angular distributions of reconstructed events with parallel tracks.

An example of a reconstructed event is shown in Fig. 10. The upper part of the figure shows a 3D reconstruction in two views. The bottom part shows the reconstruction of tracks separately according to the data of each chamber.
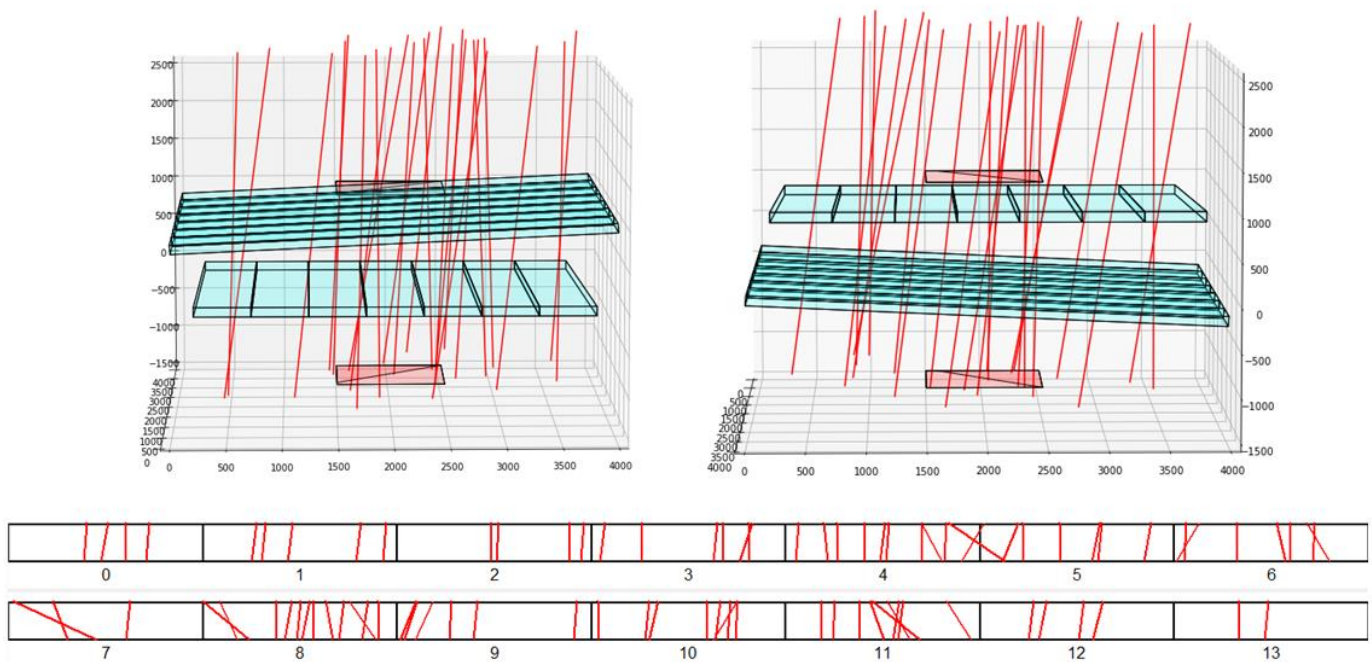

Figure 10. Example of multi-particle event reconstruction.

\section{Summary}

The new detector ProtoTREK based on drift chambers is described. The first results of registration of extensive air showers by the installation and their reconstruction using machine learning methods have been obtained. This method allows us to reconstruct and investigate events with a multiplicity of tracks up to 100 per installation plane. For vertical showers, this is approximately 10 particles per sq. $\mathrm{m}$.

Acknowledgements: The work has been performed at the Unique Scientific Facility "Experimental complex NEVOD" with the support of the Ministry of Science and Higher Education of the Russian Federation, Project "Fundamental problems of cosmic rays and dark matter", No 0723-2020-0040, support of the Russian Foundation for Basic Research within the framework of scientific project No. 20-32-90155, and support of the grant of the President of the Russian Federation MK-4137.2021.1.2. 


\section{References}

[1] E.A. Zadeba et al., J. Instrum. 9 C08018 (2014).

[2] V.V. Kindin et al., Instruments and Experimental Techniques. 61649 (2018).

[3] A.A. Petrukhin, Nucl. Instrum. Meth. Phys. Res. A. 742228 (2014).

[4] V.S. Vorobyev et al., Phys. of Atomic Nuclei. 81132 (2018).

[5] L.S. Barabash et al., Proceed. Int.Conf. Neutrino-82. 2249 (1982).

[6] V.S. Vorobev et al., J. Instrum. 15 C08007 (2020).

[7] Terasic, DE0-Nano-SoC Development Kit, https://www.terasic.com.tw/ (29 June 2021).

[8] Arduino, https://www.arduino.cc/ (29 June 2021).

[9] V.S. Vorobyev et al., J. Phys.: Conf. Ser. 945012027 (2018).

[10] C. Nebauer, IEEE Transactions on Neural Networks. 9685 (1998).

[11] S. Hochreiter et al., Neural Computation. 91735 (1997).

[12] S. Farrell et al, EPJ Web of Conferences. 15000003 (2017). 